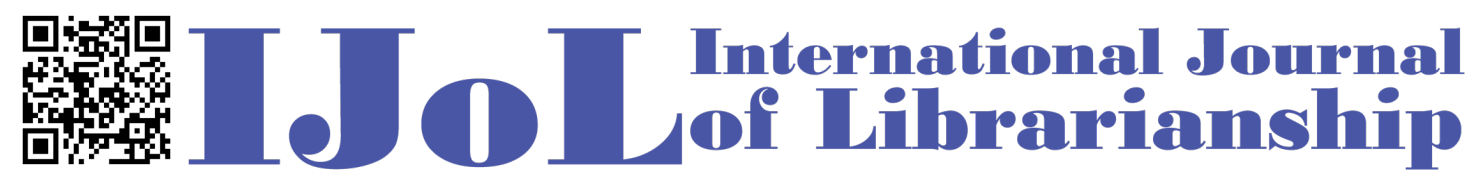

ISSN: 2474-3542 Journal homepage: https://journal.calaijol.org

\title{
Cornell Library FOLIO Case Study
}

\author{
Jenn Colt \\ Debra Howell
}

\begin{abstract}
:
Cornell University is a private university with a public mission as the Land Grant institution for New York State. After 20 years using the vended application Voyager by Ex Libris as the Library's integrated library system (ILS), on July 1, 2021 Cornell University Library completed their migration to the open-source platform, FOLIO. This case study chronicles the library's journey from FOLIO's selection to go-live.
\end{abstract}

To cite this article:

Colt, M., \& Howell, D. (2021). Cornell Library FOLIO Case Study. International Journal of Librarianship, 6(2), 13-20. https://doi.org/10.23974/ijol.2021.vol6.2.205

To submit your article to this journal:

Go to https://ojs.calaijol.org/index.php/ijol/about/submissions 


\title{
Cornell Library FOLIO Case Study
}

\author{
Jenn Colt, Cornell University \\ Debra Howell, Cornell University
}

\begin{abstract}
Cornell University is a private university with a public mission as the Land Grant institution for New York State. After 20 years using the vended application Voyager by Ex Libris as the Library's integrated library system (ILS), on July 1, 2021 Cornell University Library completed their migration to the open-source platform, FOLIO. This case study chronicles the library's journey from FOLIO's selection to go-live.
\end{abstract}

Keywords: Cornell Library, Case Study, FOLIO

\section{INTRODUCTION}

Cornell University is a private university with a public mission. With a student body of about 25,000, Cornell is the federal land-grant institution of New York State, a private endowed university, a member of the Ivy League/Ancient Eight, and a partner of the State University of New York. Cornell Library supports the university's mission with 20 different physical and digital libraries, a collection of 8.5 million volumes and 1.7 million e-books, and about 400 staff.

After 20 years using the vended application Voyager by Ex Libris as the Library's integrated library system (ILS), on July 1, 2021 Cornell Library completed our migration to the open-source platform, FOLIO, which is an acronym for the future of libraries is open.

\section{Path to FOLIO}

Cornell began exploring options for migrating away from our Voyager library management system in the early 2010s. A committee led by then Associate University Librarian (AUL) for Information Technology and Chief Technology Strategist, Dean Krafft, worked with our colleagues at Columbia University and analyzed the available ILS environments. This was known as the 2CUL project. 2CUL also included additional visionary ideas such as sharing collection development and technical services integration (Harcourt \& Leblanc, 2017). We wanted a system that would facilitate deep collaboration and push us in new directions. After evaluating Alma, we decided we wanted to avoid a single vendor dependency which led us to look at Kuali Open Library Environment (OLE). We determined that OLE best fit our commitment to open source and our hopes for having direct engagement with development. Shortly after we decided to join Kuali OLE, the community made the important decision to radically change the project's direction and break off from the Kuali platform and rewrite the code base. This change created FOLIO, which is an 
acronym for The Future of Libraries Is Open. Thusly, Cornell Library became involved in the FOLIO community in its inception in 2016.

Jason Kovari (personal communication, August 30, 2021), Director of Cataloging and Metadata Services, notes "We decided to implement OLE due to a belief in open source. We were tired of vendor lock-in and even more tired of having a lack of agency in the development of the system that is so critical to functions across our library. Further, we saw this as a means to support an ever-evolving system that will develop as our needs develop. FOLIO is built on this ethos. We did not decide to implement FOLIO for any one set of functionalities; instead, we selected FOLIO so that we can decide the direction of development along with an amazing community of peers."

Soon after FOLIO was envisioned, Cornell began dedicating development effort along with other staff and financial resources to helping stand up FOLIO on its own. The biggest factors in our continued efforts to develop and implement FOLIO are the open access vision; micro-services architecture; core library management functionality; high volume production workflows; the ability to access and modify source code; wide metadata options: MAchine-Readable Cataloging (MARC), non-MARC, linked data, etc.; and integration of E-Resource Management functionality.

\section{IMPLEMENTATION}

After several years of FOLIO development, in 2019 Cornell created a team to manage our implementation of FOLIO with the goal of going live in July 2020. We created an Implementation Team with membership from each key area across the library including; reporting, finance, metadata management, access services, user testing, acquisitions, serials processing, cataloging, eResource management (ERM), training, infrastructure, integrations, discovery, and data migration. In addition to a "lead" from each of these areas, many additional library staff became subject matter experts (SMEs), helped with training, or participated in FOLIO community special interest groups (SIGS). The library also hired an information technology project manager to lead the project.

One of the first decisions we needed to make was to determine whether we would contract with a vendor for hosting or whether we would host FOLIO ourselves. Given appropriate resources, a library can host, maintain, and upgrade FOLIO themselves. However, it became apparent that we did not have adequate staffing to self-host. After some analysis, Cornell chose EBSCO for hosting and migration services. Our EBSCO implementation consultants were invaluable partners during our migration and implementation. One of the greatest benefits of FOLIO is that it is completely customizable through its settings, and one of the most difficult things about FOLIO is that it is completely customizable through its settings. Our implementation consultant provided support, guidance, and solutions throughout the process helping us to choose the features, settings, and option that were optimal for our operations.

Implementing a system that is in development has its own set of challenges to manage. Success depended upon FOLIO development staying on track. We realized that by December of 2019, we would need to decide whether or not a July 2020 go-live was possible. FOLIO development uses Jira to track all open features, so we created a Jira dashboard which was critical to managing our implementation. The Jira dashboard displayed features we had ranked as needing at go live, one quarter after go live, and two quarters after go live. In order to evaluate our July 2020 go live decision, we went through all of the features that we ranked as needed at go live and determined whether or not they were a showstopper or if the feature was something we could live without for a short time or find a workaround for. Our guiding principle was that we could handle 
some hardships on the staff side but all patron-facing functions must work. In December 2019, we had identified 22 showstopper features that would not be available by July 2020, and we made the decision to delay one year to July 2021.

In parallel, Cornell was also migrating our ERM system from ProQuest Intota to FOLIO ERM. This project, led by Peter McCracken, Acquisitions and E-Resource Strategy Librarian and SME Emma Raub, Electronic Resources Librarian, at Cornell, was able to continue to move forward because it was standalone although it would fully integrate into FOLIO when it became available. FOLIO ERM was successfully launched at Cornell in January of 2020.

After the decision to postpone our go live until 2021 was made, we focused most of our efforts on our showstoppers. Many of our showstoppers were included in FOLIO planned development. We tracked these features closely and advocated for their importance if it seemed they might get reprioritized. For other showstoppers, we had to get creative. We partnered with the University of Chicago, Texas A\&M University, and Duke University for the development of the Online Computer Library Center (OCLC)-Single-Record-Import functionality. For our automated feed to our bursar system and Circulation Log exports, we contracted for their development and contributed the code back to the FOLIO community.

Cornell Library has customized integrations to many other applications including:

\begin{tabular}{|l|l|}
\hline Function & Product \\
\hline University Financial System & Kuali (open source) \\
\hline Cornell Bursar System & Blacklight (open source) \\
\hline Discovery and Access & BorrowDirect and ILLiad \\
\hline Interlibrary Loan & $\begin{array}{l}\text { Global Online Bibliographic Information } \\
\text { (GOBI) }\end{array}$ \\
\hline Library Technical Services & EBSCO Discovery Service (EDS) \\
\hline Library Technical Services & CaiaSoft \\
\hline Library Annex & Ares \\
\hline Course Reserves & Aeon \\
\hline Rare and Manuscript Collections & ArchiveSpace \\
\hline Rare and Manuscript Collections & In-house custom developed apps \\
\hline Library Technical Services (LTS) Workflows & \\
\hline
\end{tabular}

Many of these integrations needed to be developed. Some were developed by the FOLIO project, others were developed by vendors, and some were developed internally. We had allotted a significant amount of time for testing our integrations. However, the release version of FOLIO that we were planning to go live with was delayed for two months. This left us with about three weeks to set up our integrations and test them. As a result, a few of our integrations were not working when we cutover. For example, BorrowDirect worked for borrowing but not for lending. We completed work on our integrations within a few weeks after going live.

In March of 2020, the COVID-19 global pandemic sent most library staff home for the following year. The rest of our FOLIO implementation and go-live cutover would take place remotely or, in some cases, in a hybrid environment. Because FOLIO is a global community, in many cases we were already used to working remotely. In other areas, like training and staff engagement, we felt a deep impact.

We began a layered approach to training leading up to go live beginning in February 2021 taking into account the need for most of our training to be virtual. As part of each release cycle, 
the FOLIO development community hosts a Bug Fest. During Bug Fest all features part of the release are tested. Cornell used Bug Fests as a way to get staff involved in FOLIO. Many of our early Bug Fest testers have become our FOLIO subject matter experts who help others in the library with issues and questions. We created a training team who hosted FOLIO demos and functionspecific training sessions all of which had to be done over Zoom due to the pandemic. Each session was recorded and made available online. We created Confluence pages with FAQs and Fun Facts and sent weekly emails highlighting project news and available training. Finally, we made the decision to stop most circulation functions 10 days before go live to allow Access Services staff the opportunity to pause, rest, and take uninterrupted time to train on FOLIO.

\section{Data Migration}

Work on data migration began about two years before Cornell went live with FOLIO. We took a team-oriented approach, with the lead for data migration serving as part of Cornell's larger implementation team. Two programmers worked on the data migration team as well, one focused on bibliographic data, and one focused on acquisitions, user, and circulation data. Because Cornell worked with EBSCO as a hosting provider we were able to focus on extracting data from Voyager and then mapping it to FOLIO, while EBSCO managed the process of loading the data into FOLIO, which they were able to do more efficiently thanks to their direct access to the FOLIO database.

Here we outline several takeaways and lessons learned from the data migration efforts during our project.

\section{Begin with data clean up}

Early in the migration process, a group in library technical services was charged to begin data clean up. That group focused on cleaning up bibliographic, holding, and item records. Because FOLIO has a facet-based navigation and search system in inventory, cleaning up the bibliographic and holdings data in order to increase its searchability and improve its representation in the FOLIO inventory was important.

While the bibliographic information represented the largest pool of data that needed to be cleaned up, efforts were undertaken in other areas as well. The finance office simplified the library's fund structure, public services streamlined circulation rules, and acquisitions cleaned up unnecessary purchase orders. These clean-up efforts afforded additional benefits and opportunities. For instance, merging individual selector funds into team funds as part of the financial clean up allowed CUL more flexibility in moving allocations.

\section{Work closely with consumers of the data}

The Cornell University data migration team met regularly with departments throughout the library to learn about their work. While user experience may not seem like part of data migration, researching thoroughly the workflows and behaviors of the people using the data enabled wellinformed decisions about how the data should migrate within the FOLIO schema.

Understanding the workflows that the data will support helps to make determinations of what data to migrate and in what form. By engaging with the users extensively we also encouraged their exploration of FOLIO. We helped them to understand what their work would be like in the new system, which then allowed them to give us better information about their data needs. 
In addition to uncovering what data would need to migrate and where, we also discussed with our users what data did not need to migrate. For instance, in acquisitions we decided not to migrate closed purchase orders, which decreased the amount of data that needed to migrate and cut down the amount of clean up that was needed. For circulation data, we migrated only open loans and those with fines or fees.

Because the circulation rules in FOLIO were going to be greatly streamlined from those in Voyager, the migration team also worked to understand how the pieces of data that make up circulation rules, like loan policies and item types, would need to be changed during the migration in order to make the FOLIO circulation rules work.

During the course of this work, participation in the FOLIO community was also invaluable. We participated in the data migration special interest group and organized several conversations with other FOLIO libraries migrating from Voyager. The FOLIO community was a key support for the success of our data migration, just as it was for other areas of the project.

\section{Document data mappings}

Data migration naturally requires a lot of documentation. We created spreadsheets that mapped where the data from Voyager would migrate to in FOLIO.

For our mappings we kept a spreadsheet of each entity in FOLIO and then mapped the data elements from Voyager fields into FOLIO fields. We also documented how each data point would be extracted from Voyager. This was needed because while some fields were part of a single entity in FOLIO, they were drawn from multiple entities in Voyager. There were also fields that did not exist at all in Voyager, and so their content had to be inferred based on a variety of data points in Voyager in order to be mapped into FOLIO.

\section{Plan for multiple iterations of the migration}

We began testing migrations of our data into FOLIO months before we went live. Doing this allowed us to uncover problems in the data that we might not have found. Migrating our own data into FOLIO was also a key part of helping staff understand what using FOLIO would be like and it allowed them to see holes or problems in the data.

Once our hosting provider set up a sandbox that our staff could access. We loaded our data into the sandbox and then asked staff to test and examine it, and give us feedback. While our own reporting and logging kept us informed of patterns and problems in the data, manual examination of the data by real users proved invaluable in discovering problems and finding data points that were overlooked in the initial mapping.

\section{Provide reporting and logging on migrations}

EBSCO provided us with reporting on each iteration of the migration which let us fix problems as they arose. The logging needs to have information that will allow you to track the data through the extract, transform, and load process. We made sure to embed identifiers from Voyager throughout the data in FOLIO so that we could both maintain relationships within the data and track problems that may have occurred.

Each iteration of the migration produced a great deal of data that had to be analyzed and then evaluated for action. In some cases, the log indicated a bug within the FOLIO software that 
needed to be reported and dealt with in the FOLIO community. In other cases, the logging would indicate problems in the data from Voyager that needed to be corrected. And in yet others, data points that FOLIO considered required but that Voyager did not provide needed to be generated for the purposes of migration. In some areas a default value was necessary for required fields when Voyager did not provide a needed data point.

\section{Carefully plan the days leading to cutover}

Our iterative migration process allowed us the opportunity to time how long it took to load our data into FOLIO. This helped us to plan the days between turning off Voyager and turning on FOLIO. We created an order of operations that told us when to load each type data during the cutover, and made sure the data would be in place before we opened the new system for use by staff and the public. Some data migration continued even after the go-live, particularly data that wasn't part of circulation or discovery data sets.

After going live with FOLIO, we continued to migrate and clean up data for about six weeks. At the conclusion of that time, our data migration has been successful, and we are able to continue to refining our data in FOLIO. Our approach included listening carefully to the needs of FOLIO users, careful consideration of the difference between Voyager and FOLIO data models, and collaborative work with our hosting provider.

\section{PROJECT MANAGEMENT CHALLENGES AND RECOMMENDATIONS}

Communication is the most important project management tool when implementing an open source system; especially a system that is still in development and evolving. There is a need to balance complete transparency with championing the project and not bringing too much doom and gloom. The Cornell implementation project manager held monthly "FOLIO Friday" sessions which included project updates, timelines, and time for questions. We found that staff appreciated an honest assessment of the health of the project and factual details on areas of impact.

Additionally, effective risk management is essential. It may feel like you are constantly putting out fires. However, that is the nature of managing the implementation of a system that is still being developed. Having a list of features and a list of requirements to do a gap analysis against is not possible. A FOLIO implementation is a large, complex project with a lot of moving pieces. Based on our experience, we recommend fostering a decentralized yet unified approach. We established a lead for each functional area and the project manager must trust that each area lead is making the best decisions for Cornell and the community and that any major issues will be brought forward.

\section{Go-Live}

The weeks leading up to our July 1, 2021 go live were chaos. Several showstopper features were on the edge of not making it into our go live release, staff were fatigued from over a year of pandemic conditions and were feeling the weight of the ILS cutover on top of everything, and the other large libraries that were planning on going live at the same time as Cornell all decided to postpone. The implementation team continually re-evaluated our project status, and the Library Executive Group assessed impacts and provided guidance and steady leadership. 
One by one, we were able to cross off our showstoppers and complete our integrations and data migration. We stayed in continual communication with library staff through Zoom sessions, email, and a Cornell Library Slack channel.

In the end, the library's switch to FOLIO is a milestone not just for Cornell but for the global library community. Cornell is the first large research library in the world to go live with FOLIO, and others are learning from our experience. In implementing a software system of this size and complexity, we had a very smooth go-live experience. This is attributable to the dedication of our staff from every part of the library.

\section{Post-Go-Live}

Post-go live, we are focused on maintaining staff morale and ensuring all patron-facing features are functioning. We understood, accepted, and communicated going into our cutover that some staff functions would be impacted. For example, after go live, technical services staff reported that they were functioning at about $20 \%$ productivity. A few weeks later, that rose to $40 \%$ functionality. We expect with the two remaining FOLIO releases this calendar year that there will be a dramatic jump in functionality and productivity.

We track all bugs and features gaps on our "Known Issues and Statuses" Confluence page that all staff have access to in order to continue to maintain transparency. The implementation team is ramping down but continues to host weekly drop-in sessions for staff to ask questions about FOLIO. Our Cornell Library Slack channel has become a vibrant place for library staff to post questions and receive help from their colleagues.

Our main takeaways revealed during our post-go-live reflections on our implementation are the criticality of transparent communication, the importance of flexibility and iteration, and the value of having all staff in involved in the process.

As of November 2021, Cornell is still running the FOLIO Iris release, which is the release we went live on in July. We plan to upgrade to each future version of FOLIO as its released and our testing shows no critical feature bugs.

\section{Sustainability of Open-Source Library Platforms}

Community support and collaboration is vital to libraries now more than ever, especially when it comes to sharing ever-growing electronic resources, according to Simeon Warner, AUL for Information Technology. "It's essential to understand that a library like Cornell's has never operated alone," he said. "Now, FOLIO provides us a foundation to move forward in the way that libraries work together - and that's exciting."

It is possible that we may never have to migrate to another ILS as FOLIO continues to grow and evolve with us.

\section{References}

Harcourt, K., \& LeBlanc, J. (2017). Finale and Future: The 2CUL Technical Services Strategic Alliance. Library Resources \& Technical Services, 61(1), 43-55. https://doi.org/10.5860/1rts.61n 1.43 


\section{About the authors}

Jenn Colt is Head, Automation \& Metadata Systems in Library Technical Services at Cornell University Library. She has been with the library for more than fifteen years in a variety of IT and metadata roles. She has long-standing interests in improving discovery for patrons, and in making open source software communities more welcoming to newcomers.

Debra Howell is the Director of IT Operations for the University Libraries at Cornell University and has over 20 years of experience providing project management and strategic direction to all areas of information technology. Debra has an extensive background in facilitation, organizational change, teambuilding, and leadership. She received her Master of Industrial and Labor Relations degree from Cornell University in 2006. She is a certified Project Management Professional (PMP) and is the FOLIO implementation project manager for Cornell. Debra is an Office of the Secretary of Defense certified Master Trainer and serves as the Information Officer on the Board of the NYS American Council on Education Women's Network. 